Meta

Journal des traducteurs

Translators' Journal

\title{
Dans le mille
}

\section{Robert Dubuc}

Volume 19, numéro 2, juin 1974

URI : https://id.erudit.org/iderudit/002638ar

DOI : https://doi.org/10.7202/002638ar

Aller au sommaire du numéro

\section{Éditeur(s)}

Les Presses de l'Université de Montréal

\section{ISSN}

0026-0452 (imprimé)

1492-1421 (numérique)

Découvrir la revue

Citer cet article

Dubuc, R. (1974). Dans le mille. Meta, 19(2), 84-87.

https://doi.org/10.7202/002638ar

Ce document est protégé par la loi sur le droit d'auteur. L’utilisation des services d'Érudit (y compris la reproduction) est assujettie à sa politique d'utilisation que vous pouvez consulter en ligne.

https://apropos.erudit.org/fr/usagers/politique-dutilisation/ 


\section{PROBLÈMES ET SOLUTIONS}

\section{DANS LE MILIE}

"Mettre dans le mille », c'est évidemment au sens propre atteindre le point central de la cible, surmonté du chiffre magique, et, au figuré, c'est atteindre directement son but, tomber juste. Il est normal qu'en matière de planification, les mots cible, but, objectif fassent partie de l'arsenal du planificateur car, de dire P. Drucker, « un objectif, un but, une cible, une fois fixés, permettent de déterminer l'action qu'il faut entreprendre aujourd'hui pour obtenir des résultats demain ${ }^{1} »$.

Mais si la synonymie fait souvent l'affaire des stylistes, elle fait par contre le désespoir des techniciens qui aiment bien qu'à chaque désignation corresponde une notion précise et bien délimitée. Chez les spécialistes nord-américains de la planification ${ }^{2}$, ce besoin s'est affirmé depuis quelque temps déjà. Par exemple, le Glossary of Corporate Planning Terms établit en matière d'objectifs trois paliers bien différenciés. Après avoir donné au terme anglais objective une valeur générique, il s'emploie à distinguer basic objectives, goals et targets selon la nature des objectifs ou selon leur champ d'application :

\footnotetext{
Objectives or aims are of three types. Basic objectives define long-range fundamental purpose; they are not quantifiable or limited to a time period; they are broad. Goals and targets are performance objectives that measure; that expect attainment within a period. Goals are the first component of all company and functional plans. Targets generally refer to the subordinate objective within the goals that apply to departments and individuals. Both goals and targets are specific. Basic objectives reflect the chief

1. Cité par Christian Doulé, «la planification à long terme dans l'entreprise ", in : Entreprise, Paris, Ed. Entreprise, $\pi^{\circ} 865,7$ avril 1972, p. 61 .

2. Nous disons planification et non "planning " parce que la distinction qu'un certain usage européen établit entre ces deux termes ne nous apparaît pas utile. Parler de planification à l'échelle d'un pays et de planning à l'échelle d'une entreprise, n'est-ce pas le type même des distinctions oiseuses? Les techniques dans les deux cas sont les mêmes, seule l'échelle d'application varie.
} 
executive's grand design or mission, his conception of the organization's raison d'etre, of what it should work toward in light of the long-range opportunity 3.

D'ailleurs, Hill et Granger vont dans le même sens, mais en établissant seulement deux paliers de distinction : «A number of firms / use the term «objective» to apply to the broader, ongoing type of goal, such as growth on earnings per common share. These firms specifically use the term «goal» or «target» or «standard» for the quantification or measurement of the objective ${ }^{4}$.»

Ainsi, selon eux, les objectives correspondent à un niveau très général à l'orientation de l'entreprise. Ils ne sont précisés ni dans le temps ni dans la quantité. Ils se distinguent en cela des goals, targets ou standards, qui sont concrets et quantifiés et doivent être atteints à l'intérieur de certains délais.

On trouve confirmation de cette distinction chez Schiff ${ }^{5}$ et Barger ${ }^{6}$. Mais d'autres auteurs font une distinction fondée sur l'échelle d'application : les objectives s'appliquent à toute l'entreprise et les goals au niveau des services ${ }^{7}$. Enfin, selon la portée dans le temps, certains distinguent les long-range objectives des short-range objectives, les premiers étant parfois désignés par strategic objectives et les seconds par operational objectives ${ }^{8}$.

Nous nous retrouvons donc avec la gamme de termes suivante :

objectives : sens générique

basic objectives

long-range objectives

strategic objectives

objectifs d'orientation qui s'appliquent à toute l'entreprise et ne sont pas quantifiables

goals

targets

short-range objectives operational objectives

Ce tableau, contrairement au Glossary of Corporate Planning Terms, ne tient pas compte du palier intermédiaire, c'est-à-dire les objectifs fixés par fonction : vente, production, personnel, finance, etc. On peut toujours considérer ce dernier cas comme une application des objectifs immédiats.

Quelle solution faut-il adopter pour traduire en français ces réalités? Dans la langue courante, les distinctions entre objectif, but, fin et cible - si tant est qu'il y en ait - sont plutôt des vues de l'esprit que le résultat d'un constat de l'usage.

3. En collaboration, Glossary of Corporate Planning Terms, Montréal, Hydro-Québec, 1971, p. 11.

4. W.E. Hill et C.H. Granger, " Establishing Company Objectives », in : Handbook of Business Administration, New York, McGraw-Hill, 1967, p. 3-24, 3-25.

5. I.S. Schiff, " Planning Marketing Strategy ", in : ibid., p. 8-80.

6. J.P. Barger, "The Managing Process ", in :ibid., p. 1-8.

7. "In larger organisation, there are so many different activities, a number of which contribute only indirectly to primary company objectives, that no control is possible unless the goals of each department and division are spelled out in details $\%$. E. Dale, Management Theory and Practice, 2e éd., New York, McGraw-Hill, 1969, p. 319.

8. W.E. Hill et C.H. Granger, « Establishing Company Objectives », in : Handbook of Business Administration, p. 3-24, 3-25. 
Bénac ${ }^{9}$ donne à objectif un sens concret. Pour lui l'objectif est un but précis vers lequel on se dirige. But possède plutôt un sens général; il a trait au terme, à 1'aboutissement. Quant à cible, il ne note pour lui qu'un emploi stylistique : «être la cible de railleries ». Le Petit Robert ${ }^{10}$ va un peu dans le même sens en ce qui concerne objectif, mais lorsqu'il définit le mot but, il le fait équivaloir à objectif. La langue courante ne peut donc pas nous être d'un grand secours pour résoudre cette énigme.

Du côté de la langue technique de la gestion, l'acception générique du mot objectif, pour désigner toute fin qu'on se propose d'atteindre par une activité déterminée, semble passablement attestée. Tézenas parle en ce sens de « direction par objectifs», «du choix des moyens à mettre en œuvre pour atteindre les objectifs ${ }^{11} \gg$. C'est aussi en ce sens que l'emploient Christian Doulé ${ }^{12}$ et la très grande majorité de ceux qui abordent les questions de planification et d'orientation de l'entreprise. En ce sens il est un parfait jumeau de son sosie anglais objective.

Toutefois, le problème des acceptions spécifiques n'est pas résolu pour autant. Ce qu'on appelle en anglais basic objective, long-range objective ou strategic objective peut très bien correspondre à objectif général ${ }^{13}$ ou à objectif fondamental, selon la recommandation de l'étude publiée à ce sujet par l'HydroQuébec :

Il y a trois catégories d'objectifs : les objectifs fondamentaux (basic) précisent les buts qui donnent à l'entreprise son orientation générale; ils ne sont ni quantifiables ni de durée fixe; les objectifs intermédiaires (goals) marquent le point d'aboutissement des plans généraux ou fonctionnels de l'entreprise; les objectifs immédiats (targets) visent le rendement effectif à court terme d'un service (...) compte tenu des objectifs intermédiaires ${ }^{14}$.

On reconnaît bien ici les trois paliers dont faisait état l'étude anglaise, portant sur le même sujet et citée plus haut. À condition d'opter pour cette division en trois parties, cette solution apparaît comme très claire et très satisfaisante. Malheureusement la majorité des auteurs se contentent d'une division double. Il ne sert à rien de vouloir calquer la synonymie anglaise : abondance de biens nuit. Peut-être pourrait-on se borner à une double série de termes, l'une fondée sur la portée dans le temps, l'autre, sur les niveaux de planification.

Dans le premier cas, comme long terme s'oppose tout naturellement à court terme, on pourrait très bien rendre basic or long-range objective par objectif à Iong terme et goal, target or short-range objective par objectif à court terme.

Si l'on veut se fonder sur les niveaux de planification, nous aurons au palier de l'entreprise en général, les objectifs stratégiques qui tracent l'orientation générale

\footnotetext{
9. H. Bénac, Dictionnaire des synonymes, Paris, Hachette, 1969, p. 117.

10. P. Robert, Petit Robert, Paris, Société du Nouveau Littré, 1969.

11. J. Tézenas, Dictionnaire de l'organisation et de la gestion, Paris, Ed. de l'organisation, 1971 , p. 70.

12. Christian Doulé, "La planification à long terme dans l'entreprise », in : Entreprise, no 865 , 7 avril 1972, p. 65.

13. Ibid., p. 64 .

14. En collaboration, Glossaire de la planification générale, Montréal, Hydro-Québec, 1971 , p. 9.
} 
de la gestion et, au palier des services, les objectifs tactiques qui eux sont essentiellement quantifiables et limités dans le temps. Cette dichotomie se fonde sur le fait que la tactique détermine les moyens à mettre en cuvre pour réaliser la stratégie.

Évidemment ces distinctions sont commodes mais il serait illusoire de prétendre qu'elles sont généralement reçues. Le vocabulaire de la planification à long terme est très flottant. Les efforts pour y mettre de l'ordre, pour louables qu'ils soient, n'enlèvent toutefois pas aux usagers le privilège de décider en dernier ressort.

\section{RÉSUMÉ TERMINOLOGIQUE}

1. objective

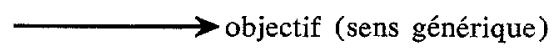

Division à trois paliers

2. basic objective

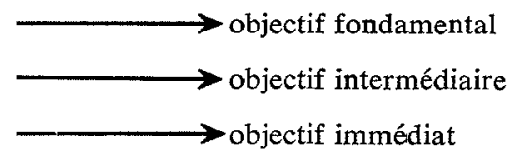

3. goal

4. target bjectif immédiat

Division à deux paliers

5. basic objective $\left.\begin{array}{l}\text { long-range objective } \\ \text { strategic objective }\end{array}\right\} \longrightarrow$ objectif stratégique (planification)

6. goal, target, standard short-range abjective objectif à court terme (temps) operational objective

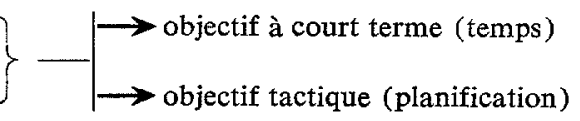

